\title{
ON THE PERIODIC SOLUTIONS PROBLEM FOR PARABOLIC INCLUSIONS WITH A LARGE PARAMETER
}

\author{
M. I. Kamenskil - P. Nistri - P. ZeCCA
}

\section{Introduction}

In this paper we study the dependence on a parameter of the periodic solutions of a system of parabolic inclusions of the form

$$
y_{i}^{\prime}(t) \in A_{i} y_{i}+f_{i}\left(t, y_{1}, \ldots, y_{n}\right), \quad i=1, \ldots, n,
$$

where $X_{1}, \ldots, X_{n}$ are separable Banach spaces, $A_{i}$ are the generators of analytic semigroups $e^{A_{i} t}$ in $X_{i}, i=1, \ldots, n$, and

$$
f_{i}: \mathbb{R} \times X_{1} \times \ldots \times X_{n} \multimap X_{i}, \quad i=1, \ldots, n,
$$

are nonlinear multivalued maps which are $T$-periodic with respect to the first variable. We consider here the case when the analytic semigroups $e^{A_{i} t}$ are not compact.

We consider two different types of dependence of the right hand side of (1) on a large parameter $\mu$. In both cases the right hand side does not have a limit as $\mu \rightarrow \infty$. But we can construct a formal limit of inclusions in such a way that the vector fields whose fixed points represent the periodic solutions of the inclusions depending on the parameter and the vector fields of the limit inclusions are homotopic.

1991 Mathematics Subject Classification. Primary 35B10, 35K55, 47H09.

Research partially supported by a Research Project MURST (40\%), CNR (Italy) and RFFT, grant 96-01-00360. 
The first case is the following:

$$
y_{i}^{\prime}(t) \in A_{i} y_{i}+f_{i}\left(\mu t, y_{1}, \ldots, y_{n}\right), \quad i=1, \ldots, n .
$$

This inclusion describes the influence on the system of exterior forces of high frequency. We will provide an averaging principle for system (2). For this, we propose a method which permits formulating the averaging principle in terms of the topological index for multivalued mappings. This formulation allows us to obtain, in a topological setting, an analogue of the classical second theorem of N. N. Bogolyubov for o.d.e.'s (see e.g. [2]). Since the involved semigroups are not compact we cannot apply the topological degree theory for compact multivalued operators; indeed, we employ the degree theory for condensing operators (see [1], [3]). For parabolic equations with compact semigroups we refer to [7], [8]. With respect to the results contained in [11] and [12] we give new and more general conditions in order to guarantee that the integral operator corresponding to the periodic problem is condensing. The measure of noncompactness we use here is the one proposed in [9] for abstract parabolic differential equations with noncompact semigroups. This measure of noncompactness is not semi-additive, and so we cannot use a linear homotopy to the limit operator. We overcome this difficulty by using a linear homotopy to a compact multivalued operator which belongs to the same class of homotopy of the limit condensing operator. For recent investigations on parabolic inclusions in Banach spaces based on the degree theory for multivalued maps we refer to [10]-[12], [14] and [15].

In the second case the parameter $\mu$ can be interpreted as a large coefficient of a diffusion problem and the relative model is of the form

$$
y_{i}^{\prime}(t) \in \mu A_{i} y_{i}+f_{i}\left(t, y_{1}, \ldots, y_{n}\right), \quad i=1, \ldots, n .
$$

We consider here the case when zero is a point of the spectrum of the natural operator generated by the operators $A_{i}$ in the product space $X$. Under our conditions zero is an eigenvalue whose geometric and algebraic multiplicities coincide. In this case the limit inclusion is an ordinary differential inclusion in the finite-dimensional eigenspace corresponding to the eigenvalue zero.

\section{Definitions and preliminary results}

Let $X$ and $Y$ be topological spaces; a multivalued map $M$ from $X$ to $Y$ will be denoted by the symbol $M: X \multimap Y$.

Definition 1.1. A multivalued map $M: X \multimap Y$ is said to be upper semicontinuous (briefly u.s.c.) if the set

$$
M^{-1}(V)=\{x \in X: M(x) \subseteq V\}
$$

is open in $X$ for every open set $V \subseteq Y$. 
A multivalued map $M: X \multimap Y$ is said to be closed if its graph

$$
\operatorname{Gr} M=\{(x, y) \in X \times Y: y \in M(x)\}
$$

is a closed subset of $X \times Y$.

Let us recall the following concepts (see, e.g. [1], [3]).

Definition 1.2. Let $X$ be a Banach space. A function $\psi$ defined on the collection of all subsets of $X$ with values in some partially ordered $(\leq)$ set is called a measure of noncompactness in $X$ if

$$
\psi(\overline{\mathrm{co}} \Omega)=\psi(\Omega)
$$

for every $\Omega \in X$. A measure of noncompactness $\psi$ is called

(i) monotone if $\Omega_{0}, \Omega_{1} \in 2^{X}$ and $\Omega_{0} \subseteq \Omega_{1}$ imply $\psi\left(\Omega_{0}\right) \leq \psi\left(\Omega_{1}\right)$;

(ii) compactly invariant if $\psi(\Omega \cup K)=\psi(\Omega)$ for every compact set $K \subset X$ and $\Omega \in 2^{X}$;

(iii) regular if $\psi(\Omega)=0$ is equivalent to the relative compactness of $\Omega$;

(iv) semi-additive if $\psi\left(\Omega_{0} \cup \Omega_{1}\right)=\max \left\{\psi\left(\Omega_{0}\right), \psi\left(\Omega_{1}\right)\right\}$ for every $\Omega_{0}, \Omega_{1} \in$ $2^{X}$.

A well known example of a measure of noncompactness with properties (i)(iv) is the Hausdorff measure of noncompactness

$$
\chi(\Omega)=\inf \{\varepsilon>0: \Omega \text { has a finite } \varepsilon \text {-net }\} .
$$

We now give another example of a measure of noncompactness which we will use in the sequel.

Example 1.3. Let $X=X_{1} \times \ldots \times X_{n}$ and let $C_{T}(X)$ be the space of all $T$-periodic continuous functions with values in $X$. Consider the measure of noncompactness $\varphi$ in $C_{T}(X)$ defined by the formula

$$
\varphi(\Omega)=\left(\chi_{1}\left(\Omega_{1}(t)\right), \ldots, \chi_{n}\left(\Omega_{n}(t)\right), \lim _{\delta \rightarrow 0} \sup _{x \in \Omega} \max _{0 \leq \tau \leq \delta}\|x(t)-x(t+\tau)\|\right)
$$

where $\Omega_{i}$ is the projection of $\Omega$ into $C_{T}\left(X_{i}\right)$ and $\Omega_{i}(t)=\left\{y(t): y \in \Omega_{i}\right\}$. The values of this measure of noncompactness belong to the space $M_{T}\left(\mathbb{R}^{n}\right) \times \mathbb{R}$ where $M_{T}\left(\mathbb{R}^{n}\right)$ is the space of $T$-periodic, measurable functions with values in $\mathbb{R}^{n}$. The measure of noncompactness $\varphi$ has properties (i)-(iii), but it does not fulfill (iv). Observe that the ordering related to property (i) is defined as follows: the space $\mathbb{R}^{n}$ is ordered by the cone $\mathbb{R}_{+}^{n}$ of positive coordinates while the space $M_{T}\left(\mathbb{R}^{n}\right)$ is ordered by the cone

$$
\mathbb{K}=\left\{y: y \in M_{T}\left(\mathbb{R}^{n}\right), y(t) \in \mathbb{R}_{+}^{n} \text { a.e. }\right\},
$$

and the space $M_{T}\left(\mathbb{R}^{n}\right) \times \mathbb{R}$ is ordered by $\mathbb{K} \times[0, \infty)$. 
Let $L(E)$ be the space of all bounded linear operators defined on the Banach space $E$. The $\chi$-norm (see [1]) of the operator $B \in L(E)$ is defined as

$$
\|B\|^{(\chi)}:=\chi(B S),
$$

where $S$ is the unit sphere in $E$. We will use the following properties of the $\chi$-norm.

Lemma 1.4 (cf. [1]). If $B \in L(E)$, then

$$
\chi(B \Omega) \leq\|B\|^{(\chi)} \chi(\Omega)
$$

for every nonempty bounded set $\Omega \subseteq E$.

Lemma 1.5 (cf. [10]). Let $E$ be separable, $B \in L(E),\|B\|^{(\chi)}<1$ and the operator $I-B$ be invertible. Then

$$
\left\|(I-B)^{-1}\right\|^{(\chi)} \leq\left(1-\|B\|^{(\chi)}\right)^{-1} .
$$

Definition 1.6 (cf. [3]). Let $E$ be a Banach space, and let $U \subset E$ be a bounded, open set. Let $F: \bar{U} \multimap E$ be an u.s.c., multivalued mapping with nonempty, compact, convex values, and let $\psi$ be a measure of noncompactness in $E$. The operator $F$ is said to be $\psi$-condensing if $\Omega \subseteq \bar{U}$ and $\psi(F(\Omega)) \geq \psi(\Omega)$ imply that $\Omega$ is relatively compact.

Definition 1.7 (cf. [3]). An u.s.c. family of multivalued maps

$$
\mathcal{F}:[0,1] \times \bar{U} \multimap E
$$

with nonempty, compact, convex values is called $\psi$-condensing if $\Omega \subset \bar{U}$ and $\psi(\mathcal{F}([0,1] \times \Omega)) \geq \psi(\Omega)$ imply that $\Omega$ is relatively compact.

Proposition 1.8 (cf. [1]). Let $\psi$ be a monotone, invariant measure of noncompactness. Let $F$ be an u.s.c., $\psi$-condensing operator on $\bar{U}$ and $\widehat{F}$ be a closed, compact operator on $\bar{U}$. Then the family $\mathcal{F}(\lambda, x)=(1-\lambda) F(x)+\lambda \widehat{F}(x)$ is an u.s.c. $\psi$-condensing family.

Definition 1.9 (cf. [3]). Two $\psi$-condensing multivalued maps

$$
F_{0}, F_{1}: \bar{U} \rightarrow 2^{E}
$$

are said to be homotopic if there exists a $\psi$-condensing family (4) such that $F_{0}(\cdot)=\mathcal{F}(0, \cdot), F_{1}(\cdot)=\mathcal{F}(1, \cdot)$ and $\mathcal{F}(\lambda, \cdot)$ has no fixed points on $\partial U$ for $\lambda \in[0,1]$. The family $\mathcal{F}$ is called a homotopy. If the measure of noncompactness $\psi$ is monotone and compactly invariant and $F$ has no fixed points on $\partial U$, one can define (see [1], [3]) an integer-valued characteristic $\operatorname{deg}(I-F, U)$, which is called degree and has the following properties: 
$1^{\circ}$. Homotopic $\psi$-condensing operators have equal degrees. In the class of $\psi$-condensing operators homotopic to $F$ there exists a compact operator $\widehat{F}$ such that $\mathcal{F}(\lambda, x)=(1-\lambda) F(x)+\lambda \widehat{F}(x)$ is a homotopy.

$2^{\circ}$. Let $U_{i}, i \in \mathbb{N}$, be a family of pairwise disjoint open subsets of $U$ and suppose $F$ has no fixed points on $\bar{U} \backslash \bigcup_{i=1}^{\infty} U_{i}$. Then the degrees $\operatorname{deg}\left(I-F, U_{i}\right)$ are defined for all $i \in \mathbb{N}$; only a finite number of them are different from zero, and

$$
\operatorname{deg}(I-F, U)=\sum_{i=1}^{\infty} \operatorname{deg}\left(I-F, U_{i}\right)
$$

$3^{\circ}$. If $F(x) \equiv x_{0}$, then $\operatorname{deg}(I-F, U)= \begin{cases}1 & \text { if } x_{0} \in U, \\ 0 & \text { if } x_{0} \notin U .\end{cases}$

$4^{\circ}$. If $\operatorname{deg}(I-F, U) \neq 0$ then $F$ has at least one fixed point in $U$.

Theorem 1.10 (cf. [11]). Let $L$ be a closed subspace of $E$ and let $F: \bar{U} \multimap L$ be an u.s.c., $\psi$-condensing multivalued mapping with nonempty, compact, convex values which has no fixed points on $\partial U$. Then

$$
\operatorname{deg}_{E}(I-F, U)=\operatorname{deg}_{L}(I-F, U) .
$$

A multivalued map $G:[a, b] \multimap E$ with nonempty, compact, convex values is said to be measurable if it satisfies any of the following two equivalent conditions:

(i) the set $G^{-1}(V)=\{t \in[a, b]: G(t) \subseteq V\}$ is measurable for every open set $V \subseteq E$;

(ii) there exists a sequence $\left\{g_{n}\right\}_{n=1}^{\infty}$ of measurable functions $g_{n}:[a, b] \rightarrow E$ such that $G(t)=\overline{\left\{g_{n}(t)\right\}_{n=1}^{\infty}}$ for all $t \in[a, b]$ (see e.g. [4]).

We denote by $S_{G}^{1}$ the set of all Bochner integrable selectors of the multivalued $\operatorname{map} G:[a, b] \multimap E$, i.e.

$$
S_{G}^{1}=\left\{g \in L^{1}([a, b], E): g(t) \in G(t) \text { a.e. }\right\} .
$$

If $S_{G}^{1} \neq \emptyset$, then $G$ is called integrable and

$$
\int_{\mathcal{I}} G(s) d s:=\left\{\int_{\mathcal{I}} g(s) d s: g \in S_{G}^{1}\right\}
$$

for every measurable set $\mathcal{I} \subseteq[a, b]$.

Clearly, if $G$ is measurable and integrably bounded (i.e. there exists $\alpha \in$ $L_{+}^{1}([a, b])$ such that $\|G(t)\|:=\max \{\|y\|: y \in G(t)\} \leq \alpha(t)$ a.e. $)$, then $G$ is integrable.

We also need the following property: 
Lemma 1.11 (cf. [11]). Let $G:[a, b] \multimap E$ be a multivalued map with nonempty bounded values. Assume $G$ is integrable, integrably bounded and

$$
\chi(G(t)) \leq \gamma(t) \quad \text { a.e. on }[a, b],
$$

where $\gamma \in L_{+}^{1}([a, b])$. Then

$$
\chi\left(\int_{\mathcal{I}} G(s) d s\right) \leq \int_{\mathcal{I}} \gamma(s) d s
$$

for every measurable set $\mathcal{I} \subseteq[a, b]$. In particular, if $\chi(G(\cdot)) \in L_{+}^{1}([a, b])$, then

$$
\chi\left(\int_{\mathcal{I}} G(s) d s\right) \leq \int_{\mathcal{I}} \chi(G(s)) d s .
$$

We also recall some results from the theory of semigroups which we will use in the sequel (see e.g. [13]).

TheOREM 1.12. A closed operator $A$ is the infinitesimal generator of the analytic semigroup $e^{A t}$ if and only if the resolvent set of $A$ contains a half-plane $\operatorname{Re} \lambda \leq \sigma_{0}$ and the resolvent satisfies there the inequality

$$
\left\|(\lambda I-A)^{-1}\right\| \leq C(1+|\lambda|)^{-1} \text { for some } C>0 .
$$

If $A$ is the infinitesimal generator of the analytic semigroup $e^{A t}$, then

$$
e^{A t}=-\frac{1}{2 \pi i} \int_{\Pi(\beta, \sigma)} e^{\lambda t}(\lambda I-A)^{-1} d \lambda, \quad t>0,
$$

where $\Pi(\beta, \sigma)$ consists of the two rays

$$
\lambda=\sigma+\varrho e^{-i \beta} \quad \text { and } \quad \lambda=\sigma+\varrho e^{i \beta}, \quad \sigma \leq \sigma_{0}, \arcsin \frac{1}{C}<\beta<\frac{\pi}{2} .
$$

If $\sigma_{0}<0$, then the negative fractional powers of $A$ are defined by the formula

$$
A^{-\alpha}=-\frac{1}{2 \pi i} \int_{\Pi(\beta, \sigma)} \lambda^{-\alpha}(\lambda I-A)^{-1} d \lambda, \quad 0<\alpha<1 .
$$

The operator

$$
A^{\alpha} e^{A t}=-\frac{1}{2 \pi i} \int_{\Pi(\beta, \sigma)} \lambda^{\alpha} e^{\lambda t}(\lambda I-A)^{-1} d \lambda, \quad t>0,
$$

satisfies the estimate

$$
\left\|A^{\alpha} e^{A t}\right\| \leq C t^{-\alpha} .
$$

From this inequality one obtains

$$
\left\|A^{-\alpha}\left(e^{A t}-I\right)\right\| \leq C t^{\alpha} .
$$

In what follows, when no confusion can arise, the constants will be indicated by the same letter $C$. 


\section{The integral operator}

In this section we want to investigate the existence of periodic solutions of (1) when $f_{i}, i=1, \ldots, n$, have nonempty convex values. We study first the properties of the integral operator

$$
\begin{aligned}
\Gamma x=\left\{y: y \in C_{T}(X),\right. & y(t)=e^{A t}\left[I-e^{A T}\right]^{-1} \int_{0}^{T} e^{A(T-s)} g(s) d s \\
& \left.+\int_{0}^{t} e^{A(t-s)} g(s) d s, g \in S_{\Phi(\cdot, x(\cdot))}^{1}, g \text { is } T \text {-periodic }\right\}
\end{aligned}
$$

where

$$
\begin{aligned}
e^{A \tau} g & =\left(e^{A_{1} \tau} g_{1}, \ldots, e^{A_{n} \tau} g_{n}\right), \\
\Phi(s, y) & =\left(f_{1}\left(s, y_{1}, \ldots, y_{n}\right), \ldots, f_{n}\left(s, y_{1}, \ldots, y_{n}\right)\right) .
\end{aligned}
$$

For one parabolic inclusion this operator was considered in [11], [12].

We assume the following conditions on the operators $f_{i}$ and $A_{i}, i=1, \ldots, n$ :

(F1) $f_{i}$ is $T$-periodic in the first variable, i.e.

$$
f_{i}(t+T, \cdot) \equiv f_{i}(t, \cdot), \quad t \in \mathbb{R}
$$

(F2) for every $x \in X$ the multivalued map $f_{i}(\cdot, x):[0, T] \rightarrow \operatorname{Kc}\left(X_{i}\right)$ admits a measurable selector. Here $\operatorname{Kc}\left(X_{i}\right)$ denotes the collection of all nonempty, compact, convex subsets of $X_{i}$;

(F3) for almost all $t \in[0, T]$, the multivalued map $f_{i}(t, \cdot): X \rightarrow \operatorname{Kc}\left(X_{i}\right)$ is u.s.c.;

(F4) there exists an $(n \times n)$-matrix $M$ with nonnegative components $\left(m_{i j}\right)$ such that

$$
\chi_{i}\left(f_{i}\left([0, T] \times D_{1} \times \ldots \times D_{n}\right)\right) \leq \sum_{j=1}^{n} m_{i j} \chi_{j}\left(D_{j}\right)
$$

for every bounded $D_{1} \subset X_{1}, \ldots, D_{n} \subset X_{n}$. Here $\chi_{i}$ denotes the Hausdorff measure of noncompactness in the space $X_{i}, i=1, \ldots, n$;

(A) for any $i=1, \ldots, n$, the closed linear operator $A_{i}$ defined on the separable Banach space $X_{i}$ generates the analytic semigroup $e^{A_{i} t}$ satisfying

$$
\left\|e^{A_{i} t}\right\|^{(\chi)} \leq e^{-\gamma_{i}(t-s)}
$$

where $\gamma_{i}>0$ and the zero solution of the system of ordinary differential equations

$$
z_{i}^{\prime}=-\gamma_{i} z_{i}+\sum_{j=1}^{n} m_{i j} z_{j}, \quad i=1, \ldots, n,
$$

is exponentially stable. 
Without loss of generality we can assume that the semigroup

$$
e^{A t} x=\left(e^{A_{1} t} x_{1}, \ldots, e^{A_{n} t} x_{n}\right)
$$

in the space $X$ satisfies the inequality

$$
\left\|e^{A t}\right\| \leq C e^{-d t},
$$

where $d>0$ and $C \geq 1$. In order to obtain this estimate, it is sufficient to subtract and add to the right hand side of every inclusion (1) the term $L y_{i}$ with the constant $L$ sufficiently large and to consider as new $A_{i}$ the operator $A_{i}-L I$ and as new $f_{i}$ the multivalued map $f_{i}\left(t, y_{1}, \ldots, y_{n}\right)+L y_{i}$. It is easy to see that for the new $A_{i}$ and $f_{i}$ conditions (A), (F1)-(F4) and the estimate (12) are still satisfied. Observe that (12) implies $1 \notin \sigma\left(e^{A T}\right)$ and so $0 \notin \sigma(A)$.

From condition (F4) it follows that $f_{i}$ is bounded on every bounded set and so every measurable selector from (F2) is integrable. As in [12] one can prove the following result.

Proposition 2.1. For every sequence $\left\{x_{m}\right\}$ of continuous functions, where $x_{m} \in C([0,1], X)$, uniformly convergent to $x_{0}$, the sequence of selectors $g_{m}(s) \in$ $f_{i}\left(s, x_{m}(s)\right)$ is weakly compact in $L^{1}\left([0, T], X_{i}\right)$ and its limit points are selectors of $f_{i}\left(\cdot, x_{0}(\cdot)\right)$.

Following the lines of [12] it is easy to see that the integral operator $\Gamma$ is u.s.c. The following theorem shows that it is also condensing with respect to the measure of noncompactness $\varphi$ given in Example 1.3.

THEOREM 2.2. Let conditions (F1)-(F4) and (A) be satisfied. Then $\Gamma$ is a $\varphi$-condensing operator.

To prove the theorem we need the following lemma.

Lemma 2.3. Let $v \in C_{T}\left(\mathbb{R}^{n}\right)$. Assume that its components $v_{i}(t), i=$ $1, \ldots, n$, satisfy the following integral inequalities:

$$
\begin{aligned}
v_{i}(t) \leq & e^{-\gamma_{i} t}\left(I-e^{-\gamma_{i} T}\right)^{-1} \int_{0}^{T} e^{-\gamma_{i}(T-s)} \sum_{j=1}^{n} m_{i j} v_{j}(s) d s \\
& +\int_{0}^{t} e^{-\gamma_{i}(t-s)} \sum_{j=1}^{n} m_{i j} v_{j}(s) d s, \quad i=1, \ldots, n,
\end{aligned}
$$

where $\gamma_{i}$ and $m_{i j}$ are the constants of condition (A). Then $v(t) \equiv 0$. 
Proof. Let $u(t)=\left(u_{1}(t), \ldots, u_{n}(t)\right)$ be the function in $C_{T}\left(\mathbb{R}^{n}\right)$ defined by

$$
\begin{aligned}
u_{i}(t)= & e^{-\gamma_{i} t}\left(I-e^{-\gamma_{i} T}\right)^{-1} \int_{0}^{T} e^{-\gamma_{i}(T-s)} \sum_{j=1}^{n} m_{i j} v_{j}(s) d s \\
& +\int_{0}^{t} e^{-\gamma_{i}(t-s)} \sum_{j=1}^{n} m_{i j} v_{j}(s) d s, \quad i=1, \ldots, n .
\end{aligned}
$$

Then for $i=1, \ldots, n$, we have $v_{i}(t) \leq u_{i}(t)$. The functions $u_{i}(t)$ are continuously differentiable and

$$
u_{i}^{\prime}(t)=-\gamma_{i} u_{i}(t)+\sum_{j=1}^{n} m_{i j} v_{j}(t) \leq-\gamma_{i} u_{i}(t)+\sum_{j=1}^{n} m_{i j} u_{j}(t) .
$$

From the comparison theorem it follows that $u_{i}(t) \leq z_{i}(t)$, where $z(t)=$ $\left(z_{1}(t), \ldots, z_{n}(t)\right)$ is the solution of system (11) with the initial condition

$$
z_{i}(0)=\left(I-e^{-\gamma_{i} T}\right)^{-1} \int_{0}^{T} e^{-\gamma_{i}(T-s)} \sum_{j=1}^{n} m_{i j} v_{j}(s) d s, \quad i=1, \ldots, n .
$$

On the other hand, all solutions of (11) tend to zero as $t \rightarrow \infty$. Hence by the $T$-periodicity of $v$ we obtain the assertion.

Proof of TheOrem 2.2. Let $\Omega$ be a bounded subset of $C_{T}(X)$ such that

$$
\varphi(\Omega) \leq \varphi(\Gamma \Omega)
$$

where the ordering $(\leq)$ is the one defined in Example 1.3. Note that the set $\Gamma \Omega$ is bounded and equicontinuous (see [11]). Then $\Omega$ is equicontinuous and therefore the functions $\chi_{i}\left(\Omega_{i}(\cdot)\right), i=1, \ldots, n$, are continuous. From (11), (14) and Lemmas 1.5 and 1.11, we have

$$
\begin{aligned}
& \chi_{i}\left(\Omega_{i}(t)\right) \leq \chi_{i}\left(\left\{e^{A_{i} t}\left[I-e^{A_{i} T}\right]^{-1} \int_{0}^{T} e^{A_{i}(T-s)} g_{i}(s) d s\right.\right. \\
& \left.\left.\quad+\int_{0}^{t} e^{A_{i}(t-s)} g_{i}(s) d s, g_{i} \in S_{f_{i}(\cdot, \Omega(\cdot))}^{1}, g_{i} \text { is } T \text {-periodic }\right\}\right) \\
& \leq e^{-\gamma_{i} t}\left(I-e^{-\gamma_{i} T}\right)^{-1} \int_{0}^{T} e^{-\gamma_{i}(T-s)} \chi_{i}\left(f_{i}\left(s, \Omega_{1}(s), \ldots, \Omega_{n}(s)\right)\right) d s \\
& \quad+\int_{0}^{t} e^{-\gamma_{i}(t-s)} \chi_{i}\left(f_{i}\left(s, \Omega_{1}(s), \ldots, \Omega_{n}(s)\right)\right) d s \\
& \leq e^{-\gamma_{i} t}\left(I-e^{-\gamma_{i} T}\right)^{-1} \int_{0}^{T} e^{-\gamma_{i}(T-s)} \sum_{j=1}^{n} m_{i j} \chi_{j}\left(\Omega_{j}(s)\right) d s \\
& \quad+\int_{0}^{t} e^{-\gamma_{i}(t-s)} \sum_{j=1}^{n} m_{i j} \chi_{j}\left(\Omega_{j}(s)\right) d s, i=1, \ldots, n .
\end{aligned}
$$


Since the functions $v_{i}(t)=\chi_{i}\left(\Omega_{i}(t)\right)$ satisfy the conditions of Lemma 2.3, we have $\chi_{i}\left(\Omega_{i}(t)\right) \equiv 0$. So by the Arzelà criterion we have the relative compactness of $\Omega$.

\section{Averaging principle}

In this section we consider the system (2) where the operators $f_{i}$ and $A_{i}$ satisfy conditions (F1)-(F4) and (A).

If we consider the change of variables $\tau=\mu t, x(\tau)=y\left(\mu^{-1} \tau\right)$, then the problem of finding $(T / \mu)$-periodic solutions of $(2)$ is equivalent to the problem of finding $T$-periodic solutions of the system

$$
x_{i}^{\prime}(t) \in \varepsilon A_{i} x_{i}+\varepsilon f_{i}\left(t, x_{1}, \ldots, x_{n}\right), \quad i=1, \ldots, n,
$$

where $\varepsilon=1 / \mu$.

Denote by $f_{i}^{0}$ the multioperator defined by the formula

$$
f_{i}^{0}(v)=\frac{1}{T} \int_{0}^{T} f_{i}(s, v) d s .
$$

Clearly, $f_{i}^{0}: X \rightarrow \mathrm{Kc}\left(X_{i}\right)$. In fact, a convex combination of selectors is also a selector. Moreover, if $y_{m} \in f_{i}^{0}(v)$ and $y_{m} \rightarrow y_{0}$, then the sequence of selectors $g_{m}$ such that

$$
y_{m}=\frac{1}{T} \int_{0}^{T} g_{m}(s) d s
$$

is relatively weakly compact by Proposition 2.1. Therefore if a subsequence $\left\{g_{m_{k}}\right\}$ weakly converges to $g \in S_{f(\cdot, v)}^{1}$, then

$$
\frac{1}{T} \int_{0}^{T} g_{m_{k}}(s) d s \stackrel{w}{\longrightarrow} \frac{1}{T} \int_{0}^{T} g(s) d s
$$

and so $y_{0}=\frac{1}{T} \int_{0}^{T} g(s) d s$.

Lemma 3.1. The operator $f_{i}^{0}$ defined in (16) is u.s.c. on $X$.

Proof. Assume by contradiction that $f_{i}^{0}$ is not u.s.c. at some point $v_{0}$. Then there exist $v_{m} \rightarrow v_{0}$, a $2 d$-neighbourhood $V_{2 d}$ of $f_{i}^{0}\left(v_{0}\right)$ and $\left\{y_{m}\right\}$ such that $y_{m} \in f_{i}^{0}\left(v_{m}\right)$ and $y_{m} \notin V_{2 d}$ for any $m \in \mathbb{N}$. Because the sequence $\left\{y_{m}\right\}$ is relatively compact and $f_{i}^{0}\left(v_{0}\right)$ is closed and convex, without loss of generality, we can assume that there exists a functional $\ell$ such that

$$
\left\langle\ell, y_{m}\right\rangle \geq d \quad \text { and } \quad\left\langle\ell, f_{i}^{0}\left(v_{0}\right)\right\rangle \leq 0
$$

Consider a sequence $\left\{g_{m}(s)\right\}$ of selectors of $f_{i}\left(s, v_{m}\right)$ such that

$$
y_{m}=\frac{1}{T} \int_{0}^{T} g_{m}(s) d s
$$


and $\left\{g_{m_{k}}\right\}$ is a subsequence weakly convergent to $g$. Then

$$
\frac{1}{T} \int_{0}^{T} g(s) d s=y \in f_{i}^{0}\left(v_{0}\right)
$$

and

$$
\frac{1}{T} \int_{0}^{T}\left\langle\ell, g_{m_{k}}(s)-g(s)\right\rangle d s \rightarrow 0 \quad \text { as } n \rightarrow \infty .
$$

On the other hand, from (16) we have

$$
\frac{1}{T} \int_{0}^{T}\left\langle\ell, g_{m}(s)-g(s)\right\rangle d s=\left\langle\ell, y_{m}\right\rangle-\langle\ell, y\rangle \geq d>0
$$

which is a contradiction. This concludes the proof.

Observe that by Lemma 1.11,

$$
\chi_{i}\left(f_{i}^{0}\left(D_{1} \times \cdots \times D_{n}\right)\right) \leq \sum_{j=1}^{n} m_{i j} \chi_{j}\left(D_{j}\right) .
$$

Thus the operators $f_{i}^{0}, i=1, \ldots, n$, satisfy assumptions (F1)-(F4) for an arbitrary $T$. Observe that any constant function with value in $f_{i}^{0}(v)$ is a selector of $f_{i}^{0}(v)$.

Denote by $\Phi_{0}$ the operator defined by

$$
\Phi_{0}(y)=\left(f_{i}^{0}\left(y_{1}, \ldots, y_{n}\right), \ldots, f_{n}^{0}\left(y_{1}, \ldots, y_{n}\right)\right) .
$$

Let us consider the operator $A^{-1} \Phi_{0}$. If we take as selectors the constant functions as described above, then it is easy to see that

$$
\begin{aligned}
A^{-1} \Phi_{0}(v)=\left\{y: y \in C_{T}(X), y(t)\right. & =e^{A t}\left[I-e^{A T}\right]^{-1} \int_{0}^{T} e^{A(T-s)} g d s \\
& \left.+\int_{0}^{t} e^{A(t-s)} g d s, g \in S_{\Phi_{0}(v)}^{1}, g \text { is constant }\right\} .
\end{aligned}
$$

So, as in Theorem 2.2, we deduce that $A^{-1} \Phi_{0}$ is $\psi$-condensing in $X$ with respect to the measure of noncompactness $\psi$ defined by

$$
\psi(\Omega)=\left(\chi_{1}\left(\Omega_{1}\right), \ldots, \chi_{n}\left(\Omega_{n}\right)\right),
$$

where $\Omega_{i}$ is the projection of $\Omega$ to $X_{i}$. Here the ordering in $\mathbb{R}^{n}$ is given by the cone $\mathbb{R}_{+}^{n}$ (see Example 1.3).

TheOREM 3.2. Let conditions (F1)-(F4) and (A) be satisfied. Suppose that the inclusion $v \in A^{-1} \Phi_{0}(v)$ has a solution $v^{*}$ and $\operatorname{deg}_{X}\left(I-A^{-1} \Phi_{0}, B_{X}\left(v^{*}, r\right)\right) \neq$ 0 for some $r$. Then, for $\varepsilon$ sufficiently small, the inclusion (15) has a T-periodic solution $x^{\varepsilon}$ such that

$$
\max _{t}\left\|x^{\varepsilon}(t)-v^{*}\right\| \leq r
$$

In order to prove this theorem, we need the following lemma. 
Lemma 3.3. Assume that the infinitesimal generator $A$ of the analytic semigroup $e^{A t}$ satisfies (10). Let $\varepsilon_{m} \rightarrow 0$, let $\left\{g_{m}\right\}, g_{m} \in L^{1}([0, T], X)$, be a sequence weakly convergent to $g$ and suppose the set

$$
\left\{g_{m}(s): s \in[0, T], m=1,2, \ldots\right\}
$$

is bounded. Then

$$
\varepsilon_{m}\left(I-e^{\varepsilon_{m} A T}\right)^{-1} \int_{0}^{T} e^{\varepsilon_{m} A(T-s)} g_{m}(s) d s \stackrel{w}{\longrightarrow} A^{-1} \frac{1}{T} \int_{0}^{T} g(s) d s \quad \text { as } m \rightarrow \infty .
$$

Proof. We have to prove that for every functional $\ell \in X^{*}$ we have

$$
\begin{aligned}
\left\langle\ell, \varepsilon_{m}\left(I-e^{\varepsilon_{m} A T}\right)^{-1} \int_{0}^{T} e^{\varepsilon_{m} A(T-s)}\right. & \left.g_{m}(s) d s\right\rangle \\
& \rightarrow\left\langle\ell, A^{-1} \frac{1}{T} \int_{0}^{T} g(s) d s\right\rangle \text { as } m \rightarrow \infty .
\end{aligned}
$$

For this, for a given $\nu>0$ we choose $d>0$ such that

$$
\left|\left\langle\ell, \varepsilon_{m}\left(I-e^{\varepsilon_{m} A T}\right)^{-1} \int_{T-d}^{T} e^{\varepsilon_{m} A(T-s)} g_{m}(s) d s\right\rangle\right|<\frac{\nu}{3}, \quad m=1,2, \ldots,
$$

and

$$
\left|\left\langle\ell, A^{-1} \frac{1}{T} \int_{T-d}^{T} g(s) d s\right\rangle\right|<\frac{\nu}{3} .
$$

This is possible since the set in (18) is bounded, $\left\|e^{\varepsilon_{m} A(T-s)}\right\| \leq C$ for some $C>0$ and

$$
\left\|\varepsilon_{m}\left(I-e^{\varepsilon_{m} A T}\right)^{-1}\right\| \leq \varepsilon_{m}\left(1+C \sum_{k=1}^{\infty} e^{-\varepsilon_{m} k d T}\right) \leq C \varepsilon_{m} \sum_{k=0}^{\infty} e^{-\varepsilon_{m} k d T} \leq 2 C /(d T) .
$$

Moreover, for any $\alpha \in(0,1)$,

$$
\varepsilon_{m}\left(I-e^{\varepsilon_{m} A T}\right)^{-1} A^{\alpha} e^{\varepsilon_{m} A(T-s)} \rightarrow \frac{1}{T} A^{-1+\alpha}, \quad s \in[0, T-d],
$$

where the convergence is in the $L(X)$-norm. In fact,

$$
\begin{aligned}
& \varepsilon_{m}\left(I-e^{\varepsilon_{m} A T}\right)^{-1} A^{\alpha} e^{\varepsilon_{m} A(T-s)} \\
& \quad=-\frac{1}{2 \pi i} \int_{\Pi(\beta, \sigma)} \varepsilon_{m}\left(1-e^{\varepsilon_{m} \lambda T}\right)^{-1} \lambda^{\alpha} e^{\varepsilon_{m} \lambda(T-s)}(\lambda I-A)^{-1} d \lambda \\
& \quad=\frac{1}{T}\left(-\frac{1}{2 \pi i} \int_{\Pi(\beta, \sigma)} \varepsilon_{m} \lambda T\left(1-e^{\varepsilon_{m} \lambda T}\right)^{-1} e^{\varepsilon_{m} \lambda(T-s)} \lambda^{-1+\alpha}(\lambda I-A)^{-1} d \lambda\right),
\end{aligned}
$$

and the last integral converges uniformly, since the function

$$
\varepsilon_{m} \lambda T\left(1-e^{\varepsilon_{m} \lambda T}\right)^{-1} e^{\varepsilon_{m} \lambda(T-s)}
$$


is uniformly bounded for $s \in[0, T-d]$ and the resolvent $(\lambda I-A)^{-1}$ satisfies (5). Therefore, one can pass to the limit under the integral obtaining (19). Furthermore, by (19) and the boundedness of the set defined by (18) we have

$$
\begin{aligned}
&\left\langle\left(A^{-\alpha}\right)^{*} \ell, \varepsilon_{m}\left(I-e^{\varepsilon_{m} A T}\right)^{-1} \int_{0}^{T-d} A^{\alpha} e^{\varepsilon_{m} A(T-s)} g_{m}(s) d s\right. \\
&\left.-A^{-1+\alpha} \frac{1}{T} \int_{0}^{T-d} g_{m}(s) d s\right\rangle \rightarrow 0,
\end{aligned}
$$

and so we can choose $m_{1}$ in such a way that the absolute value of this term is less than $\nu / 6$ for $m>m_{1}$. Furthermore, since $g_{m} \stackrel{w}{\rightarrow} g$ we obtain

$$
\left\langle\left(A^{-\alpha}\right)^{*} \ell, A^{-1+\alpha} \frac{1}{T} \int_{0}^{T-d} g_{m}(s) d s-A^{-1+\alpha} \frac{1}{T} \int_{0}^{T-d} g(s) d s\right\rangle \rightarrow 0,
$$

and so we can choose $m_{2}>m_{1}$ such that for $m>m_{2}$ the inequality

$$
\left|\left\langle\left(A^{-\alpha}\right)^{*} \ell, A^{-1+\alpha} \frac{1}{T} \int_{0}^{T-d} g_{m}(s) d s-A^{-1+\alpha} \frac{1}{T} \int_{0}^{T-d} g(s) d s\right\rangle\right| \leq \frac{\nu}{6}
$$

is satisfied.

Finally, for $m>m_{2}$ we have

$$
\begin{aligned}
& \left|\left\langle\ell, \varepsilon_{m}\left(I-e^{\varepsilon_{m} A T}\right)^{-1} \int_{0}^{T} e^{\varepsilon_{m} A(T-s)} g_{m}(s) d s-A^{-1} \frac{1}{T} \int_{0}^{T} g(s) d s\right\rangle\right| \\
& \leq\left|\left\langle\ell, \varepsilon_{m}\left(I-e^{\varepsilon_{m} A T}\right)^{-1} \int_{T-d}^{T} e^{\varepsilon_{m} A(T-s)} g_{m}(s) d s\right\rangle\right|+\left|\left\langle\ell, A^{-1} \frac{1}{T} \int_{T-d}^{T} g(s) d s\right\rangle\right| \\
& \quad+\mid\left\langle\left(A^{-\alpha}\right)^{*} \ell, \varepsilon_{m}\left(I-e^{\varepsilon_{m} A T}\right)^{-1} \int_{0}^{T-d} A^{\alpha} e^{\varepsilon_{m} A(T-s)} g_{m}(s) d s\right. \\
& \left.\quad-A^{-1+\alpha} \frac{1}{T} \int_{0}^{T-d} g(s) d s\right\rangle \mid \\
& \quad+\left|\left\langle\left(A^{-\alpha}\right)^{*} \ell, A^{-1+\alpha} \frac{1}{T} \int_{0}^{T-d} g_{m}(s) d s-A^{-1+\alpha} \frac{1}{T} \int_{0}^{T-d} g(s) d s\right\rangle\right| \leq \nu .
\end{aligned}
$$

Proof of Theorem 3.2. Since $A^{-1} \Phi_{0}$ is a $\psi$-condensing operator, there exists (see property $1^{\circ}$ of Definition 1.9 ) a compact operator $\widehat{A^{-1} \Phi_{0}}$ such that the following condition is satisfied:

(LH) the linear homotopy $\lambda A^{-1} \Phi_{0}(v)+(1-\lambda) \widehat{A^{-1} \Phi_{0}}(v)$ has no fixed points on $\partial B_{X}\left(v^{*}, r\right)=S_{X}\left(v^{*}, r\right)$.

Consider in $C_{T}(X)$ the operator which associates with every $x \in C_{T}(X)$ the set of constant functions $\widehat{A^{-1} \Phi_{0}}(x(0))$. This operator is compact. We now prove that the integral operator $\Gamma_{\varepsilon}$, associated with (15), is linearly homotopic to $\widehat{A^{-1} \Phi_{0}}(x(0))$ on $S_{C_{T}}\left(v^{*}, r\right)$ for $\varepsilon$ sufficiently small. Assume the contrary. Then 
there are sequences $\varepsilon_{m} \rightarrow 0, \lambda_{m} \in[0,1], \lambda_{m} \rightarrow \lambda_{0}$ and $x_{m} \in S_{C_{T}}\left(v^{*}, r\right)$ such that

$$
x_{m} \in \lambda_{m} \Gamma_{\varepsilon_{m}} x_{m}+\left(1-\lambda_{m}\right) \widehat{A^{-1} \Phi_{0}}\left(x_{m}(0)\right) .
$$

Or equivalently,

$$
\begin{aligned}
x_{m}(t) & =\lambda_{m} \varepsilon_{m} e^{\varepsilon_{m} A t}\left(I-e^{\varepsilon_{m} A T}\right)^{-1} \int_{0}^{T} e^{\varepsilon_{m} A(T-s)} g_{m}(s) d s \\
& +\lambda_{m} \varepsilon_{m} \int_{0}^{t} e^{\varepsilon_{m} A(t-s)} g_{m}(s) d s+\left(1-\lambda_{m}\right) w_{m},
\end{aligned}
$$

where $g_{m} \in S_{\Phi\left(\cdot, x_{m}(\cdot)\right)}^{1}, g_{m}$ is $T$-periodic and $w_{m} \in \widehat{A^{-1} \Phi_{0}}\left(x_{m}(0)\right)$. The sequence $\left\{w_{m}\right\}$ is relatively compact.

We want to prove that the sequence $\left\{x_{m}\right\}$ is relatively compact. For this, we first prove that

$$
\left\|x_{m}(t)-x_{m}(0)\right\| \rightarrow 0 \quad \text { as } m \rightarrow \infty, \text { whenever } t \in[0, T] .
$$

Since the functions $x_{m}$ are $T$-periodic it is sufficient to estimate the difference $x_{m}(t)-x_{m}(0)$ only for $t \in[0, T]$. We have

$$
\begin{aligned}
x_{m}(t)-x_{m}(0) & =\lambda_{m} \varepsilon_{m}\left(e^{\varepsilon_{m} A t}-I\right)\left(I-e^{\varepsilon_{m} A T}\right)^{-1} \int_{0}^{T} e^{\varepsilon_{m} A(T-s)} g_{m}(s) d s \\
& +\lambda_{m} \varepsilon_{m} \int_{0}^{t} e^{\varepsilon_{m} A(t-s)} g_{m}(s) d s, \quad t \in[0, T] .
\end{aligned}
$$

For the second term on the right hand side we have

$$
\left\|\lambda_{m} \varepsilon_{m} \int_{0}^{t} e^{\varepsilon_{m} A(t-s)} g_{m}(s) d s\right\| \leq C T \varepsilon_{m} \rightarrow 0 \quad \text { as } m \rightarrow \infty .
$$

For the first term, if we put $N=\left[1 / \varepsilon_{m}\right]$ and $M=\sup _{m} \sup _{s}\left\|g_{m}(s)\right\|$ we obtain

$$
\begin{aligned}
\left\|\varepsilon_{m}\left(e^{\varepsilon_{m} A t}-I\right)\left(I-e^{\varepsilon_{m} A T}\right)^{-1} \int_{0}^{T} e^{\varepsilon_{m} A(T-s)} g_{m}(s) d s\right\| \\
=\left\|A^{-\alpha}\left(e^{\varepsilon_{m} A t}-I\right) \varepsilon_{m} \sum_{k=0}^{\infty} e^{k \varepsilon_{m} A T} \int_{0}^{T} A^{\alpha} e^{\varepsilon_{m} A(T-s)} g_{m}(s) d s\right\| \\
\leq\left(\varepsilon_{m} t\right)^{\alpha}\left(\left\|\varepsilon_{m} \sum_{k=0}^{N} \int_{0}^{T} A^{\alpha} e^{\varepsilon_{m} A(T(k+1)-s)} g_{m}(s) d s\right\|\right. \\
\\
\left.+\left\|\varepsilon_{m} \sum_{k=0}^{\infty} e^{k \varepsilon_{m} A T} \int_{0}^{T} A^{\alpha} e^{\varepsilon_{m} A(T(N+2)-s)} g_{m}(s) d s\right\|\right) \\
\leq\left(\varepsilon_{m} T\right)^{\alpha}\left(C M \varepsilon_{m} \sum_{k=0}^{N} \int_{0}^{T} \frac{d s}{\varepsilon_{m}^{\alpha}(T(k+1)-s)^{\alpha}}\right.
\end{aligned}
$$




$$
\begin{aligned}
& \left.+\frac{C \varepsilon_{m}}{1-e^{-\varepsilon_{m} d T}} \int_{0}^{T} \frac{d s}{\left(\varepsilon_{m} T(N+1)\right)^{\alpha}}\right) \\
\leq & \left(\varepsilon_{m} T\right)^{\alpha} C M\left(\frac{\varepsilon_{m}^{1-\alpha}}{1-\alpha} \sum_{k=0}^{N}\left((T(k+1))^{1-\alpha}-(T k)^{1-\alpha}\right)\right. \\
& \left.+\frac{2}{d\left(\varepsilon_{m} T(N+1)\right)^{\alpha}}\right) \\
\leq & \left(\varepsilon_{m} T\right)^{\alpha} C(\alpha) M\left(\left(\varepsilon_{m}(N+1)\right)^{1-\alpha}+\left(\varepsilon_{m}(N+1)\right)^{-\alpha}\right) \\
\leq & 3 C(\alpha) M\left(\varepsilon_{m} T\right)^{\alpha} \rightarrow 0 \text { as } m \rightarrow \infty
\end{aligned}
$$

The relation (20) implies that

$$
\chi_{i}\left(\left\{\left(x_{m}(t)\right)_{i}\right\}\right) \equiv \chi_{i}\left(\left\{\left(x_{m}(0)\right)_{i}\right\}\right), \quad i=1, \ldots, n .
$$

As the functions $x_{m}$ are $T$-periodic,

$$
x_{m}\left(\left(\left[1 / \varepsilon_{m}\right]+1\right) T\right)=x_{m}(0)
$$

Therefore,

$$
\begin{aligned}
& \lambda_{m} \varepsilon_{m} e^{\varepsilon_{m} A\left(\left[1 / \varepsilon_{m}\right]+1\right) T}\left(I-e^{\varepsilon_{m} A T}\right)^{-1} \int_{0}^{T} e^{\varepsilon_{m} A(T-s)} g_{m}(s) d s \\
& \quad+\lambda_{m} \varepsilon_{m} \int_{0}^{\left(\left[1 / \varepsilon_{m}\right]+1\right) T} e^{\varepsilon_{m} A\left(\left(\left[1 / \varepsilon_{m}\right]+1\right) T-s\right)} g_{m}(s) d s+\left(1-\lambda_{m}\right) w_{m} \\
& =\lambda_{m} \varepsilon_{m}\left(I-e^{\varepsilon_{m} A T}\right)^{-1} \int_{0}^{T} e^{\varepsilon_{m} A(T-s)} g_{m}(s) d s+\left(1-\lambda_{m}\right) w_{m},
\end{aligned}
$$

since

$$
\begin{aligned}
& \left(I-e^{\varepsilon_{m} A T}\right)^{-1} \int_{0}^{T} e^{\varepsilon_{m} A(T-s)} g_{m}(s) d s \\
& =\left(I-e^{\varepsilon_{m} A\left(\left[1 / \varepsilon_{m}\right]+1\right) T}\right)^{-1} \int_{0}^{\left(\left[1 / \varepsilon_{m}\right]+1\right) T} e^{\varepsilon_{m} A\left(\left(\left[1 / \varepsilon_{m}\right]+1\right) T-s\right)} g_{m}(s) d s .
\end{aligned}
$$

Finally,

$$
\begin{aligned}
x_{m}(t)= & \lambda_{m} \varepsilon_{m} e^{\varepsilon_{m} A T}\left(I-e^{\varepsilon_{m} A\left(\left[1 / \varepsilon_{m}\right]+1\right) T}\right)^{-1} \\
& \cdot \int_{0}^{\left(\left[1 / \varepsilon_{m}\right]+1\right) T} e^{\varepsilon_{m} A\left(\left(\left[1 / \varepsilon_{m}\right]+1\right) T-s\right)} g_{m}(s) d s \\
& +\lambda_{m} \varepsilon_{m} \int_{0}^{t} e^{\varepsilon_{m} A(T-s)} g_{m}(s) d s+\left(1-\lambda_{m}\right) w_{m}
\end{aligned}
$$


Making the change of variables $x_{m}\left(\tau / \varepsilon_{m}\right)=v_{m}(\tau), \varepsilon_{m} s=\xi$, we obtain

$$
\begin{aligned}
v_{m}(\tau)= & \lambda_{m} e^{A \tau}\left(I-e^{\varepsilon_{m} A\left(\left[1 / \varepsilon_{m}\right]+1\right) T}\right)^{-1} \\
& \cdot \int_{0}^{\varepsilon_{m}\left(\left[1 / \varepsilon_{m}\right]+1\right) T} e^{A\left(\left(\left[1 / \varepsilon_{m}\right]+1\right) T-\xi\right)} \widetilde{g}_{m}(\xi) d \xi \\
& +\lambda_{m} \int_{0}^{\tau} e^{A(\tau-\xi)} \widetilde{g}_{m}(\xi) d \xi+\left(1-\lambda_{m}\right) v_{m}
\end{aligned}
$$

where $\widetilde{g}_{m}(\xi) \in \Phi\left(\xi / \varepsilon_{m}, v_{m}(\xi)\right)$.

From (20) we have

$$
\left\|v_{m}(\tau)-v_{m}(0)\right\|=\left\|x_{m}\left(\tau / \varepsilon_{m}\right)-x_{m}(0)\right\| \text { too } \quad \text { as } m \rightarrow \infty .
$$

And so from (21),

$$
\chi_{i}\left(\left\{\left(v_{m}(\tau)\right)_{i}\right\}\right) \equiv \chi_{i}\left(\left\{\left(v_{m}(0)\right)_{i}\right\}\right)=\chi_{i}\left(\left\{\left(x_{m}(0)\right)_{i}\right\}\right), \quad i=1, \ldots, n .
$$

In order to estimate $\chi_{i}\left(\left\{\left(v_{m}(\tau)\right)_{i}\right\}\right)$, we now prove that the sequence $\left\{B_{m} w\right\}$ defined by

$$
B_{m} w=\left(I-e^{\varepsilon_{m} A\left(\left[1 / \varepsilon_{m}\right]+1\right) T}\right)^{-1} \int_{0}^{\varepsilon_{m}\left(\left[1 / \varepsilon_{m}\right]+1\right) T} e^{A\left(\left(\left[1 / \varepsilon_{m}\right]+1\right) T-\xi\right)} w(\xi) d \xi
$$

converges in norm to

$$
B w=\left(I-e^{A T}\right)^{-1} \int_{0}^{T} e^{A(T-\xi)} w(\xi) d \xi
$$

In fact, let $\varepsilon_{m}\left(\left[1 / \varepsilon_{m}\right]+1\right)=1+\varrho \varepsilon_{m}$, where $\varrho \in[0,1]$, and consider

$$
\begin{aligned}
\| B_{m} w- & B w \| \\
\leq & \left\|\left(I-e^{A T\left(1+\varrho \varepsilon_{m}\right)}\right)^{-1}\right\| \cdot \|\left(e^{A T\left(1+\varrho \varepsilon_{m}\right)}-e^{A T} \|\right. \\
& \cdot\left\|\left(I-e^{A T}\right)^{-1}\right\|\left\|\int_{0}^{T\left(1+\varrho \varepsilon_{m}\right)} e^{A\left(T\left(1+\varrho \varepsilon_{m}\right)-\xi\right)} w(\xi) d \xi\right\| \\
+ & \left\|\left(I-e^{A T}\right)^{-1}\right\|\left\|\int_{T}^{T\left(1+\varrho \varepsilon_{m}\right)} e^{A\left(T\left(1+\varrho \varepsilon_{m}\right)-\xi\right)} w(\xi) d \xi\right\| \\
& +\left\|\left(I-e^{A T}\right)^{-1}\right\| \\
& \cdot \int_{0}^{T}\left\|A^{\alpha} e^{A(T-\xi)}\right\| \cdot\left\|A^{-\alpha}\left(e^{A \varrho \varepsilon_{m}}-I\right)\right\| \cdot\|w(\xi)\| d \xi .
\end{aligned}
$$

All the terms on the right hand side of (23) tend to zero since

$$
\left\|e^{A T\left(1+\varrho \varepsilon_{m}\right)}-e^{A T}\right\| \rightarrow 0 \quad \text { as } m \rightarrow \infty,
$$

and

$$
\left\|\int_{T}^{T\left(1+\varrho \varepsilon_{m}\right)} e^{A\left(T+\left(1+\varrho \varepsilon_{m}\right)-\xi\right)} w(\xi) d \xi\right\| \leq C \varrho \varepsilon_{m}\|w\|
$$


and from (6), (7) we have

$$
\int_{0}^{T}\left\|A^{\alpha} e^{A(T-\xi)}\right\| \cdot\left\|A^{-\alpha}\left(e^{A \varrho \varepsilon_{m}}-I\right)\right\| \cdot\|w(\xi)\| d \xi \leq C T^{\alpha}\left(C \varrho \varepsilon_{m}\right)^{\alpha}\|w\| .
$$

Let us note now that

$$
\begin{aligned}
\chi_{i}\left(\left\{\left(\lambda_{m} \widetilde{g}_{m}(\xi)\right)_{i}\right\}\right) & \leq \chi_{i}\left(\left\{\left(\lambda_{m} \Phi\left(\xi / \varepsilon_{m}, v_{m}(\xi)\right)\right)_{i}\right\}\right) \\
& \leq \chi_{i}\left(\overline{\operatorname{co}} f_{i}\left([0, T] \times\left\{v_{m}(\xi)\right\}\right)\right)=\chi_{i}\left(f_{i}\left([0, T] \times\left\{v_{m}(\xi)\right\}\right)\right) \\
& \leq \sum_{j=1}^{n} m_{i j} \chi_{j}\left(\left\{\left(v_{m}(s)\right)_{j}\right\}\right) .
\end{aligned}
$$

On the other hand (see $(22)), \chi_{i}\left(\left\{\left(v_{m}(\tau)\right)_{i}\right\}\right)$ satisfies the following inequalities

$$
\begin{aligned}
\chi_{i}\left(\left\{\left(v_{m}(\tau)\right)_{i}\right\}\right) \leq & \chi_{i}\left(\left\{e^{A_{i} \tau}\left(I-e^{\varepsilon_{m} A_{i}\left(\left[1 / \varepsilon_{m}\right]+1\right) T}\right)^{-1}\right.\right. \\
& \cdot \int_{0}^{\varepsilon_{m}\left(\left[1 / \varepsilon_{m}\right]+1\right) T} e^{A_{i}\left(\left(\left[1 / \varepsilon_{m}\right]+1\right) T-\xi\right)} \lambda_{m}\left(\widetilde{g}_{m}(\xi)\right)_{i} d \xi \\
& \left.\left.+\int_{0}^{\tau} e^{\varepsilon_{m} A(\tau-\xi)} \lambda_{m}\left(\widetilde{g}_{m}(\xi)\right)_{i} d \xi+\left(1-\lambda_{m}\right) w_{m}\right\}\right) \\
= & \chi_{i}\left(\left\{e^{A_{i} \tau}\left(I-e^{\varepsilon_{m} A_{i} T}\right)^{-1} \int_{0}^{T} e^{A_{i}(T-\xi)} \lambda_{m}\left(\widetilde{g}_{m}(\xi)\right)_{i} d \xi\right.\right. \\
& \left.\left.+\int_{0}^{\tau} e^{A(\tau-\xi)} \lambda_{m}\left(\widetilde{g}_{m}(\xi)\right)_{i} d \xi\right\}\right) \\
\leq & e^{-\gamma_{i} t}\left(I-e^{-\gamma_{i} T}\right)^{-1} \int_{0}^{T} e^{-\gamma_{i}(T-s)} \sum_{j=1}^{n} m_{i j} \chi_{j}\left(\left\{\left(v_{m}(s)\right)_{j}\right\}\right) d s \\
& +\int_{0}^{t} e^{-\gamma_{i}(t-s)} \sum_{j=1}^{n} m_{i j} \chi_{j}\left(\left\{\left(v_{m}(s)\right)_{j}\right\}\right) d s, \quad i=1, \ldots, n .
\end{aligned}
$$

Since the functions $\chi_{i}\left(\left\{\left(v_{m}(\tau)\right)_{i}\right\}\right), i=1, \ldots, n$, satisfy the conditions of Lemma 2.3, we have $\chi_{i}\left(\left\{\left(v_{m}(\tau)\right)_{i}\right\}\right) \equiv 0, i=1, \ldots, n$. Therefore, from (22), $\chi_{i}\left(\left\{\left(x_{m}(0)\right)_{i}\right\}\right)=0, i=1, \ldots, n$, and in virtue of $(20)$ the sequence $\left\{x_{m}\right\}$ is relatively compact. Without loss of generality, we may suppose that

$$
x_{m} \rightarrow x_{0} \in S_{C_{T}}\left(v^{*}, r\right) \quad \text { and } \quad w_{m} \rightarrow w_{0} \quad \text { as } m \rightarrow \infty .
$$

Then from (20), $x_{0}$ is a constant function and $x_{0} \in S_{X}\left(v^{*}, r\right)$. As already noticed in the proof of Proposition 2.1, the sequence $\left\{g_{m}\right\}$ is weakly compact. Assume that $\left\{g_{m}\right\}$ weakly converges to some $g_{0}$; then $g_{0} \in S_{\Phi\left(\cdot, x_{0}\right)}^{1}$ (see Section 2). Since the operator $\widehat{A^{-1} \Phi_{0}}$ is closed, we have $w_{0} \in \widehat{A^{-1} \Phi_{0}}\left(x_{0}\right)$. By Lemma 3.3 we can pass to the limit in the equality

$$
x_{m}(0)=\lambda_{m} \varepsilon_{m}\left(I-e^{\varepsilon_{m} A T}\right)^{-1} \int_{0}^{T} e^{\varepsilon_{m} A(T-s)} g_{m}(s) d s+\left(1-\lambda_{m}\right) w_{m},
$$


obtaining

$$
x_{0}(0)=\lambda_{0} A^{-1} \frac{1}{T} \int_{0}^{T} g_{0}(s) d s+\left(1-\lambda_{0}\right) w_{0} .
$$

Hence, $x_{0} \in S_{X}\left(v^{*}, r\right)$ and

$$
x_{0} \in \lambda_{0} A^{-1} \Phi_{0}\left(x_{0}\right)+\left(1-\lambda_{0}\right) \widehat{A^{-1} \Phi_{0}}\left(x_{0}\right),
$$

which contradicts (LH). Therefore, by Proposition 1.8 and property $1^{\circ}$ of Definition 1.9 , for $\varepsilon>0$ sufficiently small, we obtain

$$
\operatorname{deg}_{C_{T}}\left(I-\Gamma_{\varepsilon}, B_{C_{T}}\left(v^{*}, r\right)\right)=\operatorname{deg}_{C_{T}}\left(I-A^{-1} \Phi_{0}, B_{C_{T}}\left(v^{*}, r\right)\right)
$$

and by Theorem 1.10,

$$
\operatorname{deg}_{C_{T}}\left(I-A^{-1} \Phi_{0}, B_{C_{T}}\left(v^{*}, r\right)\right)=\operatorname{deg}_{X}\left(I-A^{-1} \Phi_{0}, B_{X}\left(v^{*}, r\right)\right) \neq 0 .
$$

Finally, by property $4^{\circ}$ of Definition 1.9 , there exists a fixed point of $\Gamma_{\varepsilon}$ in $B_{C_{T}}\left(v^{*}, r\right)$ and so a $T$-periodic solution $x^{\varepsilon}$ of the inclusion (15). This concludes the proof.

\section{Inclusions with large coefficient of diffusion}

This section deals with the problem of finding $T$-periodic solutions of inclusion (3), which we rewrite in the form

$$
y^{\prime}(t) \in \mu A y+\Phi(t, y)
$$

where the operators $A$ and $\Phi$ are defined in (8) and (9).

In addition to conditions $(\mathrm{F} 1)-(\mathrm{F} 4)$ and $(\mathrm{A})$ we assume:

$\left(\mathrm{A}^{+}\right) 0 \in \sigma(A)$ and it is a simple pole of the resolvent $(\lambda I-A)^{-1}$, that is,

$$
(\lambda I-A)^{-1}=\lambda^{-1} P+P_{0}+\lambda P_{1}+\ldots,
$$

where $P$ is the Riesz projector. Furthermore,

$$
\left\|e^{A t}(I-P)\right\| \leq C e^{-d t}
$$

for some $d>0$.

From inequality (10) it follows that the projector $P$ has a finite-dimensional range and zero is an eigenvalue of $A$. The expansion of the resolvent means simply that the geometric and the algebraic multiplicities of the zero eigenvalue coincide.

Consider now the finite-dimensional inclusion

$$
x^{\prime}(t) \in P \Phi(t, x(t))
$$


where $x(t) \in P X, t \in[0, T]$, and $\Phi$ is defined by (9). A $T$-periodic solution of (26) is a solution of the integral inclusion $x \in \Gamma_{0} x$, where

$$
\begin{aligned}
& \Gamma_{0} x=\left\{y: y \in C_{T}(X), y(t)=e^{-t}\left[I-e^{-T}\right]^{-1} \int_{0}^{T} e^{-(T-s)} g(s) d s\right.
\end{aligned}
$$

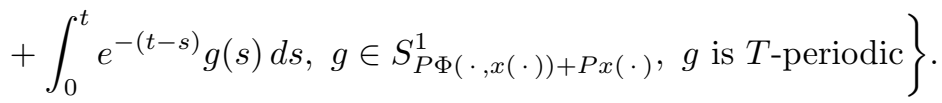

Note that $\Gamma_{0}$ is a compact operator since the projector $P$ is finite-dimensional.

Theorem 4.1. Let conditions (F1)-(F4), (A), $\left(\mathrm{A}^{+}\right)$be satisfied and assume that the inclusion (26) has a T-periodic solution $x^{0}$ such that $\operatorname{deg}(I-$ $\left.\Gamma_{0}, B\left(x^{0}, r\right)\right) \neq 0$ for some $r>0$. Then for $\mu$ sufficiently large, the inclusion (3) has a T-periodic solution $x^{\mu}$ such that

$$
\max _{t}\left\|x^{\mu}(t)-x^{0}(t)\right\| \leq r
$$

Proof. Rewrite the inclusion (24) in the form

$$
y^{\prime}(t) \in(\mu A-P) y+\Phi(t, y)+P y .
$$

The operators $\Phi+P$ and $\mu A-P$ satisfy conditions (F1)-(F4) and (A), respectively. Since $1 \notin \sigma\left(e^{(\mu A-P) T}\right)$, we can define the integral operator $\Gamma$ for $(27)$ as in Section 2, and we denote it by $\Gamma_{\mu}$. By Proposition 1.9 the family

$$
\mathcal{F}(\lambda, x)=\lambda \Gamma_{\mu} x+(1-\lambda) \Gamma_{0} x
$$

is $\varphi$-condensing. We want to prove that for $\mu$ sufficiently large, $\mathcal{F}(\lambda, x)$ is a homotopy on $S_{C_{T}}\left(x^{0}, r\right)$. Assume the contrary; then there exist sequences $\mu_{m} \rightarrow$ $\infty, \lambda_{m} \in[0,1], \lambda_{m} \rightarrow \lambda_{0}$ and $x_{m} \in S_{C_{T}}\left(x^{0}, r\right)$ such that

$$
x_{m} \in \lambda_{m} \Gamma_{\mu_{m}} x_{m}+\left(1-\lambda_{m}\right) \Gamma_{0} x_{m} .
$$

Or equivalently,

$$
\begin{aligned}
x_{m}(t)= & \lambda_{m} e^{\left(\mu_{m} A-P\right) t}\left(I-e^{\left(\mu_{m} A-P\right) T}\right)^{-1} \\
& \cdot \int_{0}^{T} e^{\left(\mu_{m} A-P\right)(T-s)} g_{m}(s) d s \\
& +\lambda_{m} \int_{0}^{t} e^{\left(\mu_{m} A-P\right)(t-s)} g_{m}(s) d s \\
& +\left(1-\lambda_{m}\right) e^{-t}\left(I-e^{-T}\right)^{-1} \int_{0}^{T} e^{-(T-s)} \widehat{g}_{m}(s) d s \\
& +\left(1-\lambda_{m}\right) \int_{0}^{t} e^{-(t-s)} \widehat{g}_{m}(s) d s,
\end{aligned}
$$

where $g_{m} \in S_{\Phi\left(\cdot, x_{m}(\cdot)\right)+P x_{m}(\cdot)}^{1}, \widehat{g}_{m} \in S_{P \Phi\left(\cdot, x_{m}(\cdot)\right)+P x_{m}(\cdot)}^{1}$ and $g_{m}, \widehat{g}_{m}$ are $T$ periodic. 
Now the sequence of operators $B_{m}$ defined by the formulas

$$
\begin{aligned}
B_{m} y(t)= & e^{\left(\mu_{m} A-P\right) t}\left(I-e^{\left(\mu_{m} A-P\right) T}\right)^{-1} \int_{0}^{T} e^{\left(\mu_{m} A-P\right)(T-s)} y(s) d s \\
& +\int_{0}^{t} e^{\left(\mu_{m} A-P\right)(t-s)} y(s) d s
\end{aligned}
$$

converges to the compact operator

$$
B_{0} y(t)=e^{-t}\left(I-e^{-T}\right)^{-1} \int_{0}^{T} e^{-(T-s)} P y(s) d s+\int_{0}^{t} e^{-(t-s)} P y(s) d s .
$$

in the operator norm. In fact, $P B_{m} y=B_{0} y$, and

$$
\begin{aligned}
\left\|(I-P) B_{m} y\right\| & \\
\leq & C\left(\int_{0}^{T-\delta}\left\|e^{\left(\mu_{m} A-P\right)(T-s)}(I-P)\right\| d s+\int_{T-\delta}^{T}\left\|e^{\left(\mu_{m} A-P\right)(T-s)}\right\| d s\right)\|y\| \\
& +C\left(\int_{0}^{t-\delta}\left\|e^{\left(\mu_{m} A-P\right)(t-s)}(I-P)\right\| d s+\int_{t-\delta}^{t}\left\|e^{\left(\mu_{m} A-P\right)(t-s)}\right\| d s\right)\|y\| .
\end{aligned}
$$

Using (25) we obtain $\left\|(I-P) B_{m} y\right\| \leq C\left(1 /\left(d \mu_{m}\right)+\delta\right)$, and from this the assertion.

Since the sequence $\left\{x_{m}\right\}$ is relatively compact, by the results of Section 2 the sequences $\left\{g_{m}\right\}$ and $\left\{\widehat{g}_{m}\right\}$ are weakly compact. Without loss of generality, we assume that

$$
\begin{aligned}
& x_{m} \longrightarrow x_{0} \in S_{C_{T}}\left(x^{0}, r\right), \\
& g_{m} \stackrel{w}{\longrightarrow} g_{0} \in S_{\Phi\left(\cdot, x_{0}(\cdot)\right)+P x_{0}(\cdot),}^{1} \\
& \widehat{g}_{m} \stackrel{w}{\longrightarrow} \widehat{g}_{0} \in S_{P \Phi\left(\cdot, x_{0}(\cdot)\right)+P x_{0}(\cdot)}^{1} .
\end{aligned}
$$

Therefore we can pass to the limit in equation (28) to obtain

$$
\begin{aligned}
x_{0}(t)= & e^{-t}\left(I-e^{-T}\right)^{-1} \int_{0}^{T} e^{-(T-s)}\left(\lambda_{0} P g_{0}(s)\left(1-\lambda_{0}\right) \widehat{g}_{0}(s)\right) d s \\
& +\int_{0}^{t} e^{-(t-s)}\left(\lambda_{0} P g_{0}(s)\left(1-\lambda_{0}\right) \widehat{g}_{0}(s)\right) d s .
\end{aligned}
$$

On the other hand, $P g_{0} \in S_{P \Phi\left(\cdot, x_{0}(\cdot)\right)+P x_{0}(\cdot)}^{1}$ and so $x_{0} \in \Gamma_{0} x_{0}$, which is a contradiction. Thus, from properties $1^{\circ}, 4^{\circ}$ of Definition 1.9 and Theorem 1.10 we obtain the assertion.

\section{REFERENCES}

[1] R. R. Akhmerov, M. I. Kamenskĭ, A. S. Potapov, A. E. Rodkina and B. N. Sadovskĭ, Measures of Noncompactness and Condensing Operators, Birkhäuser-Verlag, Basel, 1992. 
[2] N. N. Bogolyubov and Yu. A. Mitropol'skiĬ, Asymptotic Methods in the Theory of Nonlinear Oscillations, Fizmatgiz, Moscow, 1963. (Russian)

[3] Yu. G. Borisovich, B. D. Gel'man, A. D. Myshkis and V. V. Obukhovskir, Topological methods in the fixed-point theory of multivalued maps, Uspekhi Mat. Nauk 35 (1980), 59-126. (Russian)

[4] C. Castaing and M. Valadier, Convex Analysis and Measurable Multifunctions, Lecture Notes in Math., vol. 580, Springer-Verlag, Berlin, 1977.

[5] J. Diestel, Remarks on weak compactness in $L_{1}(\mu, X)$, Glasgow Math. J. 18 (1977), $87-91$.

[6] I. Ekeland and R. Temam, Convex Analysis and Variational Problems, North-Holland, Amsterdam, 1976.

[7] I. N. Gourova, An assertion of the type of the relatedness principle and second theorem of N. N. Bogolyubov in the averaging principle for parabolic equations, Qualitative and Approximate Methods for Investigating Operator Equations, Yaroslavl', 1982, pp. 47-58. (Russian)

[8] D. Henry, Geometric Theory of Semilinear Parabolic Equations, Lecture Notes in Math., vol. 840, Springer-Verlag, Berlin, 1981.

[9] M. I. Kamenskĭ Applications des opérateurs condensants au problème des solutions périodiques pour les équations paraboliques quasi-linéaires, C. R. Acad. Sci. Paris Sér. I 318 (1994), 719-722.

[10] M. I. Kamenskil̆, P. Nistri, V. V. Obukhovskĭ and P. Zecca, Optimal feedback control for a semilinear evolution equation, J. Optim. Theory Appl. 82 (1994), 503-517.

[11] M. I. KamenskiI And V. V. OBukhovskĭ, On periodic solutions of differential inclusions with unbounded operators in Banach spaces, Zb. Rad. Prirod.-Mat. Fak. Univ. u Novom Sadu Ser. Mat, 21 (1991), 173-191.

[12] Condensing multioperators and periodic solutions of parabolic functional-differential inclusions in Banach spaces, Nonlinear Anal. 20 (1993), 781-792.

[13] M. A. Krasnosel'skil̆, P. P. ZabreǏko, E. I. Pustyl'nik and P. E. Sobolevskĭ, Integral Operators in Spaces of Summable Functions, Noordhoff, Leyden, 1976.

[14] P. Nistri, V. V. OBukhovskil And P. ZeCCA, On the solvability of systems of inclusions involving noncompact operators, Trans. Amer. Math. Soc. 342 (1994), 543-562.

[15] N. S. Papageorgiou, On multivalued semilinear evolution equations, Boll. Un. Mat. Ital. B (7) 3 (1989), 1-16.

M. I. KAMENSKII

Department of Mathematics

Voronezh State University

394693, Voronezh, RUSSIA

E-mail address: kmi@mathd.vucnit.voronezh.su

P. Nistri and P. ZeCCA

Dipartimento di Sistemi e Informatica

Università di Firenze

via S. Marta n. 3

50139 Firenze, ITALY

E-mail address: pnistri@ingfi1.ing.unifi.it,pzecca@ingfi1.ing.unifi.it

TMNA : Volume $8-1996-\mathrm{N}^{\circ} 1$ 